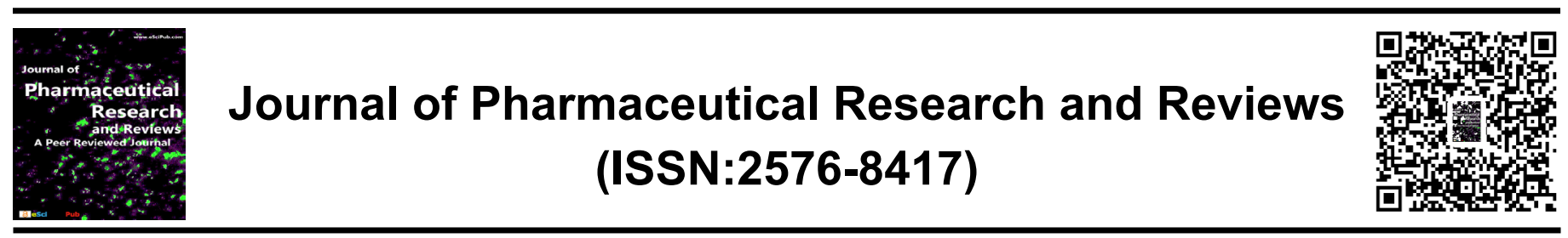

\title{
Progress in the application of LOV2 domain in protein interaction
}

\section{Xiao Shao}

College of Medicine, China Three Gorges University, Yichang, 443002, China.

\begin{abstract}
Protein-protein interactions (PPIs) and protein complexes formed by interactions are the main accomplishers of various functions ${ }^{*}$ Correspondence to Author: of cells. As an important component of biological networks, Xiao Shao protein interaction plays an important role in determining life processes such as signal transduction in cells. Although the College of Medicine, China Three Gexperimental methods for studying protein interactions are endless, there are still many shortcomings and gaps in the research on the interaction of precisely regulated protein molecules. LOV (Light Oxygen Voltage) is a light-sensitive protein domain originally discovered in the photo of plants for receiving blue light stimulation, transmitting signals, and causing a series of reactions in plants. The LOV domain belongs to a member of the PAS (Per-Arnt-Sim) superfamily and contains two subunits, LOV1 and LOV2. LOV2 is thought to cause a conformational change after receiving light stimulation, primarily responsible for activating the kinase domain. It plays an important role in orges University, Yichang, 443002, China.

intracellular signal transduction. In view of the light control and How to cite this article:

Xiao Shao. Progress in the application of LOV2 domain in protein interaction. Journal of Pharmaceutical Research and Reviews, 2019; 3:17 sensitivity of LOV2, which can be used as a research tool in protein interaction processes, the mechanism of action and structural advantages of signal transduction in LOV2 domain in protein interactions are reviewed.
\end{abstract}

Keywords: Protein interaction; LOV2 domain; Optogenetics; Light-sensitive protein; Photon 


\section{Research techniques for protein interact- ion}

Protein is a direct executor of life activities. Almost all physiological activities in the cell, including DNA replication and transcription, protein synthesis and secretion, signal transduction and metabolism, are achieved through proteins. Although many functions and behaviors in life activities are performed by a single protein, a large part requires the interaction between protein and protein, protein and nucleic acid to achieve ${ }^{[1]}$. Among them, protein-protein interaction networks and transcriptional regulatory networks are important for regulating celIs and their signals, so the study of protein-protein interactions is important. Nowadays, research methods on the mechanism of protein interaction are also emerging. The following techniques are mainly used in our experimental research: 1. Yeast two-hybrid (Y2H); 2. GST pulldown; 3. Co-IP method (Co-IP) ; 4. Cellular colocalization; 5. Phage Display Technology (PDT); 6. Fluorescence Resonance Energy Transfer Technology (FRET); 7. Bimolecular Fluorescence Complementary Technology(BIFC); 8. Surface Plasmon Resonance (SPR); 9.Protein Chip; 10. Tandem Affinity Purification (TAP); 11. Far Western blot ${ }^{[2-5]}$. These kinds of technologies have their respective focuses in the study of protein interactions. But at the same time, because of the characteristics of protein interactions, there are various deficiencies in the research process. For example, during the course of the study, when the degree of protein interaction is weak, it is not easy to be captured. But a weak, short-lived process may be the key to information transfer. Strong interactions may be more likely to be used to maintain the structure. At present, there are few studies on weak interactions, and there are also great difficulties. In addition, short-lived weak interactions are usually not the norm, so the difficulty lies in how quickly the state of protein interaction can be detected. Finally, the experimental method should reduce the false positive rate and the false negative rate as much as possible, that is, the reliability of the experimental results must be ensured.

Currently, a new cell biology tool has been developed that uses light-sensitive proteins as switching elements to accurately perturb and control the behavior of cells and organisms. The basis is the process of protein interaction, which is called "optogenetics" [6]. Because its principle is to use light control of light-sensitive proteins to regulate cell behavior and molecular signal activity, and the fluorescent effect of the tool protein in cells also brings great advantages for research. So finding a suitable light-sensitive protein is key, among the existing light-sensitive proteins, LOV is relatively small in molecular mass, soluble, and its required cofactors are present in most types of cells, and have various ways of regulating signal molecules. These characteristics make it an ideal molecule for the construction of different signal pathways for light-controlled cells ${ }^{[7]}$.

\section{Discovery of the LOV2 domain}

LOV2 was originally discovered in plant phototropin(phot), a blue light (BL) receptor widely found in plants and having two subtypes, phot1 and phot2. Phot2 works under high-radiation conditions, while phot1 responds mainly under low-irradiation conditions, both of which are related to plant phototropism, chloroplast movement, stomatal opening, and leaf opening [8]. The difference between LOV1 and LOV2 is that LOV1 plays an important role in the dimerization of receptors after blue light irradiation. LO$\mathrm{V} 2$ regulates the activity of STK. Mutation studies indicate that amino acid residues critical for phosphorylation activity of STK are near the $\mathrm{N}$-terminal region of LOV2. The regulation of STK by LOV2 has a photoreaction reaction, including the transient formation of flavin mononucleotide (FMN)-cysteinyl adduct (S390) [9]. One of the keys to the propagation of optical signals from LOV2 to STK is the conformational change of Ja-helix located downstream of the LV2C-terminal ${ }^{[10]}$. It has also been found in other studies that the LOV domain is also present in many light-sensitive proteins of eukary- 
otes and bacteria, archaea. The LOV domain can also be coupled to a number of other domains, including F-box domain, STAS domain, and zinc finger domain. This suggests that the LOV domain can also modulate the activity of these domains by absorption of blue light [11]. LOV2 belongs to a subunit of the PAS (Per-Arnt-Sim) superfamily and is a light-sensitive protein domain that acts on the interaction between proteins and proteins in signal transduction. In addition, the light sensing system ${ }^{[12]}$ also includes other families of proteins such as the BLUF (blue light sensors utilizing flavin) domain and cryptochromes (cry) ${ }^{[13]}$. Among them, LOV2 domain protein is used as a fluorescent protein, coupled with FMN, and such light absorption cofactor is present in almost all cells. Under the same conditions, its fluorescence intensity is significantly stronger than that of green fluorescent protein. Therefore, it ca$\mathrm{n}$ be regarded as a major advantage of optogenetic research tools. In addition, the LOV2 domain has a small molecular weight of only about 100-140 amino acid residues and is soluble $^{[14]}$, which is a breakthrough for protein-protein interaction studies.

\section{Important functional components for the LOV2 domain to function}

The scientists analyzed the structural features of the LOV domain by X-ray crystallography. It contains five antiparallel $\beta$-sheets and multiple $\alpha$-helices, and the FMN is surrounded by 11 conserved amino acids in the LOV domain though hydrogen bonding and intermolecular forces [15]. Both LOV1 and LOV2 undergo an independent photoperiod after exposure to blue light ${ }^{[16]}$. It involves the formation of a covalent adduct between FMN and a conserved active site cysteine residue (Cys39). LOV2 transitions from a ground state to a triplet after absorbing one photon in the blue region. The FMN then covalently combines with $\mathrm{C}(4 \mathrm{a})$ of an adjacent cysteine (Cys39) to form a emission signal in microseconds [17]. Christie JM et al. replaced alanine with Cys39 and found that it can eliminate the conduction of light signals by LOV1 and LOV2, thus failing to activate the kinase domain ${ }^{[9]}$. This illustrates the importance of Cys39 in initiating a reaction after binding to FMN. The X-ray crystal structure of the adduct with LOV showed that the cysteine shifted to $\mathrm{C}(4 \mathrm{a})$ of FMN by about $2 \mathrm{~nm}$, confirming that cysteine forms a thiol bond with the $\mathrm{C}(4 \mathrm{a})$ atom of FMN [18]. It was later discovered that the formation of a thiol bond triggered a change in the $\beta$-sheet conformation of the protein center, resulting in the separation of Ja-helix (the residue at the C-terminus of LOV2) from the surface of the $\beta$-sheet. And this signal is passed to a series of downstream domains, this step is conducive to the transmission of signals and the occurrence of autophosphorylation. It was also found in later studies that the $\mathrm{N}$-terminal linkage region of LOV2 is called $A^{\prime} \alpha / A \beta$ and interacts with Ja-helix in conformational changes ${ }^{[19]}$. Some biochemical and biophysical studies have also revealed key regions of the $\mathrm{N}$-terminal and C-terminal of LOV2.

\subsection{Ja-helix}

The key step in the transmission of optical signals from LOV2 to STK is that a conformational change occurs in Ja-helix at the C-terminus downstream of LOV2. Ja-helix runs along the surface of LOV2 and consists of approximately twenty conservative amino acids. The researchers obtained the aqueous solution structure of LOV2 and Ja-helix by means of nuclear magnetic resonance. It was found that when the hydrophobic amino acid on the hydrophobic side was replaced by a hydrophilic amino acid, the structure of Ja-helix was destroyed, indicating that the extended Ja-helix could activate the enzyme molecule ${ }^{[18]}$. Later, Ja-helix was also found in LOV2 of oatmeal phototropin, and the nuclear magnetic resonance analysis of LOV2-Ja-helix of oat phot1 showed that blue light causes the unfolding of Ja-helix, resulting in the dissociation of LOV2 and Ja-helix ${ }^{[20]}$. So the signal is passed on further. When compared with other species, the structure was found to be similar, indicating that Ja-helix plays an important role in the structure and function 
of LOV2. Recently, researchers have used time -resolved infrared spectroscopy to study the signal transduction pathway of LOV2. It is observed that the expansion of Ja-helix is divided into two steps: In the first step, the formation of the Cos 39-FMN covalent adduct occurs at 10 $\mu \mathrm{s}$, accompanied by the hydrogen bond cleavage of FMNC4 $=0$ of Gin-513, movement of the $\beta$-sheet and $\alpha$-helix; The second phase occurs approximately at $25 \mu \mathrm{s}$, and the final spectrum is approximately identical to the steady-state spectrum, indicating that the Ja-helix expansion is sufficiently complete ${ }^{[21]}$. The structural change of Ja-helix is considered to be a key process for intramolecular signal transduction from LOV2 to STK.

\section{2 $A^{\prime} \alpha / A \beta$}

$A^{\prime} \alpha / A \beta$-helix is located upstream of the N-terminus of LOV2 and interacts with Ja-helix. Kashojiya $S$ et al., by studying the LOV2-STK polypeptide from Arabidopsis phot1, found that Lys 603 and Lys475 in A'a-helix was truncated at the gap between the $A^{\prime} \alpha$ and $A \beta$ chains of LOV2 (A' $\alpha / A \beta G A P)$. When replaced with alanine, the results inhibited light-induced STK activation, but they did not affect $\mathrm{S} 390$ formation [22]. These light-induced structural changes will vary when Glu474 and Lys475 are used instead of Lys603 and Lys475, showing that the optical signal can reach Ja-helix and $A^{\prime} \alpha / A \beta$ GAP but not STK. The amino acid residues Glu474 and Lys475 in the gap are conserved in photographs of higher plants and can serve as important elements for linking Ja-helix structural changes with STK activation. A'a-helix moves along the $\beta$-fold surface of LOV2, and its edge is very close to Ja-helix, which can form a dimer, indicating a signal interaction between the two ${ }^{[23]}$.

\section{Application of LOV2 domain in protein interaction}

\subsection{LOV2 optogenetic control of enzyme fu- nction}

Recently, phage-derived anti-CRISPR (Acr) proteins have been found to naturally inhibit type II CRISPR systems, including the most widely used Streptococcus pyogenes cas9. These CRISPR antagonists also function when heterologously expressed in yeast and mammalian cells. However, the effectiveness of this approach is currently limited due to the lack of ways to easily limit Acr activity in time and space. Therefore, the scientists chose the LOV 2 domain as an accurate implementation of switching between functional Cas9 inhibitory and non-functional states under external light stimulation ${ }^{[24]}$. Insertion of the LOV2 domain into selected, surface-exposed loops of certain enzymes enables optogenetic control of enzymatic function, with the $\mathrm{N}$ and $\mathrm{C}$ ends of LOV2 very close in dark adaptation, thus after LOV2 insertion The natural enzyme conformation can be maintained.

\subsection{LOV2-based photoelectric switch tool}

The scientists designed a gene-encoded synthetic photoactivated integrin (LOVInc) [25] based on the principle of using the light-sensitive properties of the LOV2 domain as a regulatory switch. Moreover, in order to prove its wide applicability, the photo-induced recombination of multiple target proteins was monitored by a synthetic protein system. it was found that LOVInc can accurately induce protein splicing activity in mammalian cells, and its precise temporal and spatial effects depend on the recognition of specific target cells ${ }^{[26]}$.

The LOV 2 domain (AsLOV 2) encoded by the Avena Sativa Avoprotein 1 gene reversibly regulates the affinity of the polypeptide for desmin ${ }^{[28]}$.By sequence analysis and molecular modeling, the two peptides were inserted into the Ja helix of the AsLOV 2 domain while maintaining the structure of AsLOV 2 in the dark, but when Ja-helix was developed under illumination, it bound to the effector protein.Under illumination, IPAA and SsrA peptides, LOV-IPAA and LOV-SsrA bind to the target with 49-fold and 8-fold affinity, respectively. This can be demonstrated by the transcription of light-activated genes in yeast as a universal tool for lightdependent co-localization.

4.3 Control protein degradation and cellular 


\section{function}

The scientists developed a universal PSD module by combining the LOV 2 domain of Arabidopsis thaliana with the murine ornithine decarboxylase degradation sequence ODC. The production of conditional mutants and the light regulation of cyclin-dependent kinase activity have demonstrated its versatility. The PSD module applies the principle of photoregulated degradation to non-plant organisms, and it is very beneficial for controlling protein levels in biotechnology or biomedical applications and offers potential ${ }^{[29]}$. The PSD module can be ligated to the $\mathrm{C}$-terminus of the target protein localized to the cytoplasm or nucleus to obtain light control of its stability. Blue light causes a change in the LOV 2 domain, which in turn leads to activation of PSD, leading to proteasomal degradation of the entire fusion protein [30]. Next, the difference in yeast cells that produced a large amount of RFP-PSD in the presence or absence of blue light at the macroscopic level was evaluated. It was found that there was strong fluorescence in the cells in the dark, while there was no fluorescence in the cells under blue light. It indicates that the protein synthesis rate has little effect on the photoresponse of the PSD module [31].

\section{Outlook}

Light-dependent regulation represents a promising approach to controlling biological processes that provides unparalleled spatiotemporal resolution and minimal toxic effects. The regulation of proteins by light is currently the most advanced method, especially a very important tool in molecular biology. The discovery of LOV 2 provides new tools for the use of optogenetics in the field of neuroscience. There have been some studies exploring the structure and function of LOV2, which enable it to more precisely regulate specific signaling pathways within cells and reduce the interference of other signaling pathway changes. In order to be able to further leverage LOV to construct optogenetic tools that modulate more types of signaling systems, a clear understanding of the mec- hanism of action is particularly important.

\section{References}

1. Geng Guorong. Biomacromolecular Interaction [J]. Life Science, 1994(03): 1-4.

2. Wu Jian, Zhu Haixia, Zhao Zhiwei, et al. Protein Interaction Research Techniques[J].Progress in Animal Medicine, 2016, 37(02): 109-115.

3. Guo Rui, Liu Quanzhong. New Progress in Proein Interaction Research Technology[J]. Journal of Tianjin Medical University, 2015, 21(06): 542-544.

4. Shen Yaoyao, Yan Qingfeng. Advances in protein Interaction Research[J]. Life Science, 2013 25(03): 269-274.

5. Durech M, Trcka F, Vojtesek B, et al. [Methods for analysis of protein-protein and protein-ligand interactions] [J]. Klin Onkol, 2014, 27 Suppl 1: S75-S81.

6. Liu Ziqiang, Wang Wei. Techniques and applications of optogenetics[J]. Medical Review, 201 6, 22(14): 2720-2724.

7. Arinkin V, Granzin J, Rollen K, et al. Structure of a LOV protein in apo-state and implications for construction of LOV-based optical tools [J]. Sci Rep, 2017, 7: 42971.

8. Qiao Xinrong, Duan Hongbin, Ye Zhaowei. Research Progress of Plant Transluminescence Receptor and Signal Transduction Mechanism[J]. Biotechnology Bulletin, 2014(08): 1-7.

9. Christie JM, Swartz TE, Bogomolni RA, et al. Phototropin LOV domains exhibit distinct roles in regulating photoreceptor function [J].PLANT J, 2002, 32(2): 205-219.

10. Harper SM, Christie JM, Gardner KH. Disruption of the LOV-Jalpha helix interaction activates phototropin kinase activity [J]. BIOCHEMISTRY-US, 2004, 43(51): 16184-16192.

11. Crosson S, Rajagopal S, Moffat K. The LOV domain family: photoresponsive signaling modules coupled to diverse output domains[J].BIOCHEMISTRY-US, 2003, 42(1): 2-10.

12. Ziegler $\mathrm{T}$, Moglich $A$. Photoreceptor engineering [J]. Front Mol Biosci, 2015, 2: 30.

13. Song SH, Freddolino PL, Nash Al, et al. Modulating LOV domain photodynamics with a residue alteration outside the chromophore binding 
site[J].BIOCHEMISTRY-US, 2011, 50(13): 241 1-2423.

14. Yin Lengpeng. Design of fluorescent probe based on LOV2 domain and study of interaction between biliverdin and DNA. Huazhong Agricultural University; 2016. p 76.

15. Crosson S, Moffat K. Structure of a flavin-binding plant photoreceptor domain: insights into light-mediated signal transduction [J]. Proc Natl Acad Sci U S A, 2001, 98(6): 2995-3000.

16. Nakasone $\mathrm{Y}$, Ohshima M, Okajima $\mathrm{K}$, et al. Photoreaction Dynamics of LOV1 and LOV2 of Phototropin from Chlamydomonas reinhardtii [J]. J PHYS CHEM B, 2018, 122(6):1801-1815.

17. Crosson S, Moffat K. Photoexcited structure of a plant photoreceptor domain reveals a light-driven molecular switch [J].PLANT CELL, 2002, 14(5): 1067-1075.

18. Harper SM, Christie JM, Gardner KH. Disruption of the LOV-Jalpha helix interaction activates phototropin kinase activity [J]. BIOCHEMISTRY-US, 2004, 43(51): 16184-16192.

19. Aihara $Y$, Yamamoto T, Okajima K, et al. Mutations in $\mathrm{N}$-terminal flanking region of blue light-sensing light-oxygen and voltage 2 (LOV2) domain disrupt its repressive activity on kinase domain in the Chlamydomonas phototropin [J]. J BIOL CHEM, 2012, 287(13): 9901-9909.

20. Kashojiya S, Yoshihara S, Okajima K, et al. The linker between LOV2-Jalpha and STK plays an essential role in the kinase activation by blue light in Arabidopsis phototropin1, a plant blue light receptor[J].FEBS LETT, 2016 , 590(1): 139-147.

21. Konold PE, Mathes $\mathrm{T}$, Weibetaenborn $\mathrm{J}$, et al. Unfolding of the C-Terminal Jalpha Helix in the LOV2 Photoreceptor Domain Observed by Time-Resolved Vibrational Spectroscopy [J]. J PHYS CHEM LETT, 2016, 7(17): 3472-3476.

22. Kashojiya S, Okajima K, Shimada T, et al. Essential role of the A'alpha/Abeta gap in the N-terminal upstream of LOV2 for the blue light signaling from LOV2 to kinase in Arabidopsis photototropin1, a plant blue light receptor [J]. PLOS ONE, 2015, 10(4): e124284. of the LOV2 domain of the blue-light photoreceptor phototropin 1 from Arabidopsis thaliana [J]. Acta Crystallogr Sect F Struct Biol Cryst Commun, 2013, 69(Pt 12): 1316 -1321.

24. Bubeck F, Hoffmann MD, Harteveld Z, et al. Engineered anti-CRISPR proteins for optogenetic control of CRISPR-Cas9 [J]. NAT METHODS, 2018, 15(11): 924-927.

25. Qudrat A, Mosabbir A, Truong K.LOV2-Controlled Photoactivation of Protein Trans-Splicing [J]. Methods Mol Biol, 2017, 1495: 227-237.

26. Wong S, Mosabbir AA, Truong K. An Engineered Split Intein for Photoactivated Protein Trans-Splicing [J]. PLOS ONE, 2015, 10(8):e13 5965.

27. Zimmerman SP, Kuhlman B, Yumerefendi $\mathrm{H}$. Engineering and Application of LOV2-Based Photoswitches [J]. Methods Enzymol, 2016,580: 169-190.

28. Lungu OI, Hallett RA, Choi EJ, et al. Designing photoswitchable peptides using the AsLOV2 domain[J].CHEM BIOL, 2012, 19(4): 507-517.

29. Renicke C, Schuster D, Usherenko S, et al. A LOV2 domain-based optogenetic tool to control protein degradation and cellular function [J]. CHEM BIOL, 2013, 20(4): 619-626.

30. Lutz AP, Renicke C, Taxis C. Controlling Protein Activity and Degradation Using Blue Light [J]. Methods Mol Biol, 2016, 1408: 67-78.

31. Taxis C. Development of a Synthetic Switch to Control Protein Stability in Eukaryotic Cells with Light [J]. Methods Mol Biol, 2017, 1596:241-25 5.

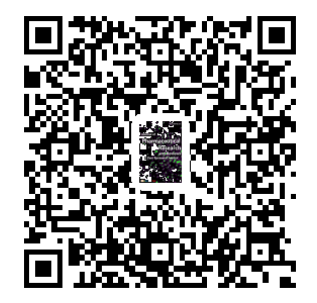

23. Halavaty AS, Moffat K. Coiled-coil dimerization 